\title{
Impact of complex NOTCH1 mutations on survival in paediatric T-cell leukaemia
}

\author{
Marcela Braga Mansur ${ }^{1}$, Rocio Hassan², Thayana C Barbosa', Alessandra Splendore ${ }^{3}$, Patricia Y Jotta ${ }^{4}$, \\ José Andrés Yunes ${ }^{4}$, Joseph L Wiemels ${ }^{5}$ and Maria S Pombo-de-Oliveira ${ }^{1,6^{*}}$
}

\begin{abstract}
Background: Molecular alterations occur frequently in T-ALL and the potential impact of those abnormalities on outcome is still controversial. The current study aimed to test whether NOTCH1 mutations and additional molecular abnormalities would impact T-ALL outcome in a series of 138 T-ALL paediatric cases.

Methods: T-ALL subtypes, status of SIL-TAL1 fusion, ectopic expression of TLX3, and mutations in FBXW7, KRAS, PTEN and NOTCH1 were assessed as overall survival (OS) and event-free survival (EFS) prognostic factors. OS and EFS were determined using the Kaplan-Meier method and compared using the log-rank test.

Results: The frequencies of mutations were $43.5 \%$ for NOTCH1, while FBXW7, KRAS and PTEN exhibited frequencies of $19.1 \%, 9.5 \%$ and $9.4 \%$, respectively. In $78.3 \%$ of cases, the coexistence of NOTCH1 mutations and other molecular alterations was observed. In multivariate analysis no statistical association was revealed between NOTCH1 mutations and any other variable analyzed. The mean length of the follow-up was 68.4 months and the OS was 50.7\%. S/LTAL1 was identified as an adverse prognostic factor. NOTCH1 mutation status was not associated with outcome, while the presence of NOTCH1 complex mutations (indels) were associated with a longer overall survival ( $p=$ 0.031) than point mutations.
\end{abstract}

Conclusion: NOTCH1 mutations alone or in combination with FBXW7 did not impact T-ALL prognosis. Nevertheless, complex NOTCH1 mutations appear to have a positive impact on OS and the SIL-TAL1 fusion was validated as a negative prognostic marker in our series of T-ALL.

\section{Background}

T-cell Acute Lymphoblastic Leukaemia (T-ALL) accounts for $\sim 15 \%$ of all childhood ALL cases, and this disease is clinically characterized as a high-risk malignancy with a relapse rate of about $30 \%$ [1,2]. T-ALL is also characterized by the occurrence of multiple genetic alterations that result in the transformation of $\mathrm{T}$-cell precursors. Distinct immunophenotypic subsets and somatic genetic alterations have been occasionally correlated with prognosis, but these results could not be consistently replicated by other studies, requiring larger number of cases to confirm associations that could support an improvement in treatment [3-6].

Since the first report addressing the role of NOTCH1 mutations in paediatric T-ALL prognosis, several

\footnotetext{
* Correspondence: mpombo@inca.gov.br

'Paediatric Haematology-Oncology Program, Research Centre, Instituto

Nacional de Câncer, Rio de Janeiro, RJ, Brazil

Full list of author information is available at the end of the article
}

controversial issues have been raised regarding the actual impact of these mutations on prognosis [7]. The NOTCH1 gene is expressed in haematopoietic stem cells (HSCs) and controls several steps of T-cell specification and differentiation. This gene was first described in a recurrent $\mathrm{t}(7 ; 9)(\mathrm{q} 34 ; \mathrm{q} 34)$ chromosomal translocation rarely found in T-ALL [8], and recently the gain-offunction NOTCH1 mutations were reported as a common event in T-ALL patients ( 50\%) [9]. These mutations mainly involve the heterodimerization (HD) domain, the C-terminal PEST/TAD domain, or both, resulting in up-regulation of Notch1 signalling $[9,10]$. However, the significance of each type of NOTCH1 mutation and especially their impact on disease recurrence remains to be investigated.

Additional molecular markers are not often included in prognostic studies limiting the evaluation of NOTCH1 mutations as an independent prognostic factor. Few studies have examined the prognostic role of 
NOTCH1 concurrently with other abnormalities [7]. Genetic lesions targeting multiple cellular pathways including T-lymphoid development, tumour suppression (FBXW7) and cell cycle regulation, as well as PI3kinase/Akt (PTEN) and Ras (KRAS) signalling appear to be central events in the pathogenesis of T-ALL [11].

Given this evidence, we hypothesized that analysis of NOTCH1 in concert with genes functionally related to Notch1 pathway, such as FBXW7, KRAS and PTEN, would provide additional relevant information regarding T-ALL prognosis. We therefore explored associations between the NOTCH1 mutations patterns and other somatic alterations in paediatric T-ALL cases in an attempt to better understand the relationship with disease progression and outcome.

\section{Methods}

\section{Subjects}

A series of 138 paediatric T-ALL were selected for this study from 178 cases, from January 2001 to January 2008, based on the availability of biological material for molecular analysis. Subjects were ascertained from four geographical regions assembled by a national network of acute leukaemia studies [12] and the majority of cases were included in a previous publication [6]. The exclusion criteria applied for the analysis was age (12 months) and the diagnosis of T-lymphoblastic lymphoma according to WHO classification $[13,14]$.

Diagnostic samples were obtained prior to any chemotherapy regimen and provided along with demographic and clinical data of each patient. Peripheral blood (PB) samples, typically with high white blood cell (WBC) count, were send along with BM aspirates. The presence or absence of blast cells in the BM aspirate and $\mathrm{PB}$ was reviewed before molecular analysis. To leukaemia diagnosis a threshold of $>20 \%$ of blast cells in the $\mathrm{BM}$ was used as lower limit for analysis. In the $\mathrm{PB}$, a clinically high WBC count or a blast percent $\geq 50 \%$ were considered suitable for our analysis.

\section{Ethical aspects}

The Ethical and Scientific Committees of the Instituto Nacional de Câncer, Rio de Janeiro, Brazil approved the study (CEP-INCA\#107/06) in accordance with the Declaration of Helsinki ethical standards.

\section{Demographic and clinical data}

Characteristics of patients obtained at diagnosis were age, gender, mediastinum involvement and elevated WBC counts. The participants' ethnicities were categorized according to the skin colour, as Whites and nonWhites. There were $60(43.5 \%)$ patients self reported as Whites and $78(56.5 \%)$ as non-Whites. In the nonWhite category blacks and all mixed-populations were included, according to criteria described elsewhere [15]. The age range was $\geq 1-18$ years-old, which was divided in two groups for data analysis as $<10$ and 10-18 yearsold. Eighty six cases (62.3\%) were from the Northeast region of Brazil, 34 (24.6\%) from the Southeast, 15 (10.9\%) cases from Central plateau; and 3 (2.2\%) from the South region.

One-hundred and one patients were treated according to Brazilian Group for Treatment of Childhood Leukaemia (GBTLI-99), while 37 were treated according to the ALL-Berlin-Frankfurt-Munster (BFM) protocols backbone strategies, as described elsewhere [16,17].

\section{Immunological characterization}

In all cases, the diagnosis was established according to criteria previously defined [13,14]. After mononuclear cell separation using Ficoll-Hypaque-Hystopaque ${ }^{\circledR}$, immunophenotype was performed in blast cells by flow cytometry using a defined panel of monoclonal antibodies, as previously described [6] and/or by immunohistochemistry methods [13,14]. Immunological classification of T-ALL was based on criteria previously published by the European Group for the Immunological Characterization of Leukaemias [18].

\section{Nucleic acids extraction and cDNA synthesis}

Genomic DNA was extracted from BM aspirate by one of the following methods: the QIAamp ${ }^{\circledR}$ DNA Blood Mini Kit (Qiagen GmbH, Hilden, Germany) ( $\mathrm{n}=78$ ), ethanol precipitation from phenol phase after RNA isolation $(n=36)$, or the salting out method $(n=24)$.

For RT-PCRs assays, the total RNA was prepared using the TRIzol reagent kit (Invitrogen, Carlsbad, California, USA) according to the manufacturer's instructions $(\mathrm{n}=129)$. cDNA was generated by reverse transcription of $2 \mu \mathrm{g}$ of total RNA using First-Strand cDNA Synthesis Kit ${ }^{\mathrm{TM}}$ (Amersham Biosciences UK Limited, Little Chalfont, UK), and stored at $-20^{\circ} \mathrm{C}$. The integrity of cDNA was examined by amplifying a fragment of the GAPDH constitutive gene, according to reported conditions [6].

\section{NOTCH1 mutations}

NOTCH1 mutations were analyzed by screening of the heterodimerization (HD) and polypeptide enriched in proline, glutamate, serine and threonine (PEST) domains according to procedures described previously [9]. In general, PEST and TAD domains are both denominated PEST domain. In summary, the N-terminal region of the HD domain of NOTCH1, encoded by exon 26, was divided in two amplicons. The amplification of exon 27, encoding the $\mathrm{C}$ terminal region of NOTCH1 HD domain, resulted in one amplicon; and sequences of exon 34 encoding the PEST domain and the contiguous 
N-region containing the TAD domain were amplified as three amplicons. All six amplicons were sequenced on both strands. Mutations were classified into two groups: complex and point mutations. Complex mutations were defined as insertions and/or deletions (also called as indels) in the gene sequence, and point mutations as single base changes. A case was considered to harbour complex mutation when an indel was detected alone or concomitant with point mutations. Classification in missense or nonsense mutations was in accordance with classical mutation definition.

\section{FBXW7 mutations}

To evaluate the mutational status of $F B X W 7$, we screened the exons 9 and 10, previously reported to be the most frequently mutated regions $[19,20]$. We performed PCR reactions for each exon with specific primers. PCR products were purified and then directly sequenced. All procedures for $F B X W 7$ screening were described elsewhere [20].

\section{SIL-TAL1 and TLX3 assays}

The SIL-TAL1 fusion and TLX3 (also known as $H O X 11 L 2)$ presence were investigated according to previously published methods $[21,22]$. PCR products of both RT-PCR assays were separated by $1.5 \%$ agarose gel electrophoresis, stained with ethidium bromide.

\section{KRAS mutations}

To study the mutations in codons 12 and 13 of the $K R A S$ gene were carried out separate PCR reactions for each codon, using 100 ng of genomic DNA. PCR products from codon 12 were digested with restriction enzyme using NE Buffer 2, $100 \mu \mathrm{g} / \mathrm{ml} \mathrm{BSA}$ and 10U of BstNI (Biolabs, New England, MA), and incubated overnight at $60^{\circ} \mathrm{C}$. The PCR products of codon 13 were digested with $2 \mathrm{U}$ of PflMI for $6 \mathrm{~h}$ at $37^{\circ} \mathrm{C}$. The digested PCR fragments were visualized on a $3 \%$ agarose gel, as previously described [23]. All the mutations found with restriction fragment length polymorphism- RFLP assay were confirmed by direct sequencing.

\section{PTEN mutations}

PTEN sequences of exons 1 and 7 were amplified by PCR. Conformational analysis was performed by heteroduplex assays, followed by direct DNA sequencing to confirm abnormal patterns, as described elsewhere [24]. PCR products were sequenced on both strands using the BigDye kit (Applied Biosystems).

\section{Statistical analysis}

Univariate $p$ values were calculated using Pearson's chisquare test. Mann-Whitney test was used to test median differences of continuous variables. Two-sided $p$ values with a significance limit of 0.05 were used throughout the study. The probability of overall survival (OS) was determined using the Kaplan-Meier method in months from the diagnosis to outcome (death, alive or last follow-up). Patients lost to follow-up were censored at their date of last known contact. Event-free survival (EFS) was the interval (in months) from diagnosis to progression at any time, relapse from complete response, or initiation of new, previously unplanned treatment or to the last follow-up in the patients with treatment success. The differences between T-ALL survival distributions were compared by the log-rank test. The multivariate Cox proportional hazard regression method was used to determine the independent prognostic factors influencing OS and EFS. Multivariate Cox analysis was performed with variables associated with a $p \leqq 0.1$ in univariate analysis such as the type of NOTCH1 mutation (point vs. complex mutations), SILTAL1 fusion and induction responses. SPSS (Statistical Product and Services Solutions, version 18.0, SPSS Inc, Chicago, IL, USA) software was used for data analyses.

\section{Results}

The main demographic, clinical, and molecular characteristics of T-ALL cases according to NOTCH1 status are shown in Table 1 . The median age of the total series of cases was 8 years (1-18 years), and a predominance of males was observed, with a 2.6:1 male/female ratio. Immunophenotyping analyses by flow cytometry were available for 130 cases, and in 8 cases the T-cell diagnosis were confirmed by immunohistochemistry with $\mathrm{CD}_{4} 5^{+} / \mathrm{CD}^{+}$. Regarding $\mathrm{T}$-cell maturation, we observed a predominance of T-IV subtype (38.0\%). Overall, 60/ 138 patients $(43.5 \%)$ presented with NOTCH1 mutations; additional molecular alterations observed were FBXW7 mutations (21/110; 19.1\%), SIL-TAL1 fusion (37/129; 28.7\%), TLX3 ectopic expression (10/119; 8.4\%), KRAS (10/105; 9.5\%) and PTEN mutations (9/96; 9.4\%). In $78.3 \%$ of cases, the coexistence of NOTCH1 mutations and other molecular alterations was observed, including FBXW7 (n = 13), SIL-TAL1 ( $\mathrm{n}=19), T L X 3(\mathrm{n}$ = 6), PTEN ( $\mathrm{n}=5)$, and KRAS ( $\mathrm{n}=4)$ mutational. However, no statistical association was disclosed between NOTCH1 mutations status and any other variable analyzed.

$n$ number of cases, mut mutated, wt wild-type, WBC white blood cells count at diagnosis. ${ }^{a} 8$ cases without information about mediastinal mass, being 4 with NOTCH1 mutation and 4 wild-type; ${ }^{\mathrm{b}}$ classification according to EGIL criteria, flow cytometry was not performed in 8 cases, 2 presented NOTCH1 mutation and 6 wild-type; ${ }^{\mathrm{c}}$ there were 22 cases without CD10 status evaluated, 7 with NOTCH1 mutation and 15 wild-type; pos, positive; neg, negative 
Table 1 Clinical, demographic and laboratorial features of T-ALL patients according to NOTCH1 status, 2001-2008

\begin{tabular}{|c|c|c|c|c|}
\hline Variable & n (\%) & $\begin{array}{c}\text { NOTCH1 mut n } \\
(\%)\end{array}$ & $\begin{array}{c}\text { NOTCH1 wt n } \\
(\%)\end{array}$ & $\begin{array}{c}p \\
\text { value }\end{array}$ \\
\hline \multicolumn{5}{|l|}{ Age (Years) } \\
\hline$<10$ & $71(51.4)$ & $28(46.7)$ & $43(55.1)$ & 0.324 \\
\hline 10-18 & $67(48.6)$ & $32(53.3)$ & $35(44.9)$ & \\
\hline \multicolumn{5}{|l|}{ Gender } \\
\hline Male & $\begin{array}{c}100 \\
(72.5)\end{array}$ & $46(76.7)$ & $54(69.2)$ & 0. 332 \\
\hline Female & $38(27.5)$ & $14(23.3)$ & $24(30.8)$ & \\
\hline \multicolumn{5}{|l|}{ WBC $\left(\times 10^{9} / \mathrm{L}\right)$} \\
\hline$<50$ & $63(45.7)$ & $29(48.3)$ & 34 (43.6) & 0.579 \\
\hline$\geq 50$ & $75(54.3)$ & $31(51.7)$ & $44(56.4)$ & \\
\hline \multicolumn{5}{|l|}{$\begin{array}{l}\text { Mediastinal } \\
\text { mass }^{a}\end{array}$} \\
\hline Yes & $58(44.6)$ & $24(42.9)$ & $34(45.9)$ & 0.726 \\
\hline No & $72(55.4)$ & $32(57.1)$ & $40(54.1)$ & \\
\hline \multicolumn{5}{|l|}{ Skin colour } \\
\hline White & $60(43.5)$ & $21(35.0)$ & $39(50.0)$ & 0.078 \\
\hline Non-white & $78(56.5)$ & $39(65.0)$ & $39(50.0)$ & \\
\hline \multicolumn{5}{|l|}{$\begin{array}{l}\text { T-ALL } \\
\text { subtypes }^{b}\end{array}$} \\
\hline $\mathrm{T}-\mathrm{I}$ & 18 (13.8) & $9(15.5)$ & $9(12.5)$ & 0.343 \\
\hline $\mathrm{T}-\|$ & $29(22.3)$ & $9(15.5)$ & $20(27.8)$ & \\
\hline $\mathrm{T}-|| \mid$ & $34(26.2)$ & $18(31.0)$ & $16(22.2)$ & \\
\hline T-IV & $49(37.7)$ & $22(37.9)$ & $27(37.5)$ & \\
\hline \multicolumn{5}{|l|}{ CD10 status $^{c}$} \\
\hline positive & $36(31.0)$ & $16(30.2)$ & $20(31.7)$ & 0.857 \\
\hline negative & $80(69.0)$ & 37 (69.8) & $43(68.3)$ & \\
\hline \multicolumn{5}{|l|}{$\begin{array}{l}\text { Molecular } \\
\text { Studies }\end{array}$} \\
\hline $\begin{array}{l}\text { SIL-TAL1 } \\
\text { pos }\end{array}$ & $37(28.7)$ & 19 (32.8) & $18(25.4)$ & 0.355 \\
\hline$\underset{\text { pos }}{\operatorname{HOX}}$ & $10(8.4)$ & $6(11.1)$ & $4(6.2)$ & 0.332 \\
\hline FBXW7 mut & $21(19.1)$ & $13(24.1)$ & $8(14.3)$ & 0.192 \\
\hline KRAS mut & $10(9.5)$ & $4(7.7)$ & $6(11.3)$ & 0.527 \\
\hline PTEN mut & $9(9.4)$ & $5(10.9)$ & $4(8.0)$ & 0.630 \\
\hline Total & $\begin{array}{c}138 \\
(100)\end{array}$ & $60(100)$ & $78(100)$ & \\
\hline
\end{tabular}

Considering that NOTCH1 and FBXW7 belong to the same signalling pathway, patients with mutations in both genes (NOTCH1 and/or FBXW7) were analyzed together as a NOTCH1-FBXW7 mutated group. In total, 62 patients (56.4\%) presented with NOTCH1-FBXW7 mutations. Patients with FBXW7, KRAS or PTEN mutations shared other molecular alterations along with NOTCH1 mutations, such as FBXW7 mutation and SIL/ TAL1 ( $\mathrm{n}=5), F B X W 7$ mutation and TLX3 $(\mathrm{n}=1)$, FBXW7 and KRAS mutations $(\mathrm{n}=2), F B X W 7$ and PTEN mutations $(\mathrm{n}=1)$. We also observed KRAS mutation and SIL/TAL1 $(\mathrm{n}=1), K R A S$ mutation and TLX3 $(\mathrm{n}=2)$, PTEN mutation and SIL-TAL1 $(\mathrm{n}=1)$, PTEN and KRAS $(\mathrm{n}=1)$, and one case exhibited PTEN, KRAS and SIL-TAL1 alterations.

The complete descriptions of NOTCH1 mutations sequences in HD and PEST domains are shown in Additional file 1: Table S1. Single nucleotide polymorphism (SNP) already described, recurrent mutations as well as previously unreported mutations were observed. Thirtyeight new mutations were found in the HD domains and 15 in PEST domain, representing 63.1\% (53/84) of all mutations found. Among these new mutations there were no SNPs, since we have checked and excluded any SNP possibility through NCBI SNP database and 1000 genomes data. The NOTCH1 SNPs found in this work have been excluded from all subsequent mutation analyses; and they were mentioned in Additional file 1: Table S1 only for descriptive purposes.

The main demographic and clinical features of $\mathrm{T}$ ALL patients with different types of NOTCH1 mutations are summarized in Table 2. Mutations were not randomly distributed, with a predominance of point mutations in the HD domain, and complex mutations in the PEST domain $(p=0.014)$. Also, nonsense mutations were more frequent in the PEST domain and missense mutations in the HD domain $(p<0.0001)$. Seven cases presented with mutations in both HD and PEST domains, with a predominance of complex mutations $(22.2 \%)$. No significant statistical differences were observed with respect to age groups, gender and $\mathrm{T}$ ALL subtypes, in respect to any type of NOTCH1 mutation. High WBC was observed in patients harbouring complex mutations, while patients with point mutations exhibited lower WBC $(p=0.035)$. Point mutations were more frequent in non-white patients $(p$ $=0.053)$; where a higher frequency of mutations in the HD domain was observed $(89.7 \%$ versus only $10.3 \%$ in PEST domain, $p=0.028$ ). Missense mutations were more frequent in $\mathrm{CD} 10+$ cases $(p=0.042)$. Eleven out of 14 nonsense mutations were caused by complex mutations, the other three by point mutations. Thirty out of 46 missense mutations were caused by point mutations and the remaining 16 by complex mutations $(p=0.004$, data not shown $)$

Overall survival of the 138 cases was $50.7 \%$ (Figures 1A). Mean length of the follow-up was 68.4 months (95\% CI 58.9-77.8 months). For 68 patients who evolved to death, the follow-up was 0-76 months (median 9), with 9 deaths occurring in the first 3 days after diagnosis and $57.4 \%$ of deaths (39 patients) occurring in the first 12 months of treatment. For 70 patients alive, the follow-up was 15-124 months (median 73.5), with 75\% of patients with 94.5 months follow-up. No differences in OS were observed in patients treated with GBTLI or BFM-based protocols (52.5\% vs. $45.9 \%$, respectively; $p=$ $0.639)$. 
Table 2 Main demographic and clinical features of T-ALL patients according to type and classification of NOTCH1 mutations

\begin{tabular}{|c|c|c|c|c|c|c|}
\hline Variables & Point Mutations ${ }^{\mathrm{a}}(\%)$ & Complex Mutations n (\%) & $p$ value & Missense n (\%) & Nonsense $\mathrm{n}(\%)$ & $p$ value \\
\hline \multicolumn{7}{|l|}{ Age (Years) } \\
\hline$<10$ & $14(42.4)$ & $14(51.9)$ & 0.466 & $21(45.7)$ & $7(50.0)$ & 0.775 \\
\hline $10-18$ & $19(57.6)$ & $13(48.1)$ & & $25(54.3)$ & $7(50.0)$ & \\
\hline \multicolumn{7}{|l|}{ Gender } \\
\hline Male & $26(78.8)$ & $20(74.1)$ & 0.668 & $34(73.9)$ & $12(85.7)$ & 0.361 \\
\hline Female & $7(21.2)$ & $7(25.9)$ & & $12(26.1)$ & $2(14.3)$ & \\
\hline \multicolumn{7}{|l|}{ WBC $\left(\times 10^{9} / L\right)$} \\
\hline$<50$ & $20(60.6)$ & $9(33.3)$ & 0.035 & $23(50.0)$ & $6(42.9)$ & 0.640 \\
\hline$\geq 50$ & $13(39.4)$ & $18(66.7)$ & & $23(50.0)$ & $8(57.1)$ & \\
\hline \multicolumn{7}{|l|}{ Skin colour } \\
\hline White & $8(24.2)$ & $13(48.1)$ & 0.053 & $15(32.6)$ & $6(42.9)$ & 0.481 \\
\hline Non-white & $25(75.8)$ & $14(51.9)$ & & $31(67.4)$ & $8(57.1)$ & \\
\hline \multicolumn{7}{|l|}{ T-ALL subtypes ${ }^{\mathrm{b}}$} \\
\hline $\mathrm{T}-\mathrm{I}$ & $4(12.9)$ & $5(18.5)$ & 0.825 & $8(17.8)$ & $1(7.6)$ & 0.589 \\
\hline $\mathrm{T}-\|$ & $4(12.9)$ & $5(18.5)$ & & $6(13.3)$ & $3(23.1)$ & \\
\hline$T-1 \|$ & $10(32.3)$ & $8(29.6)$ & & $15(33.3)$ & $3(23.1)$ & \\
\hline T-IV & $13(41.9)$ & $9(33.3)$ & & $16(35.6)$ & $6(46.2)$ & \\
\hline \multicolumn{7}{|l|}{$\mathrm{CD}_{10}$ status $^{\mathrm{C}}$} \\
\hline Positive & $11(39.3)$ & $5(20.0)$ & 0.127 & $15(37.5)$ & $1(7.7)$ & 0.042 \\
\hline Negative & $17(60.7)$ & $20(80.0)$ & & $25(62.5)$ & $12(92.3)$ & \\
\hline \multicolumn{7}{|c|}{ NOTCH1 mutated domain } \\
\hline $\mathrm{HD}$ & $28(84.8)$ & $14(51.9)$ & 0.014 & $38(82.6)$ & $4(28.6)$ & $<0.0001$ \\
\hline PEST/TAD & $4(12.1)$ & 7 (25.9) & & $4(8.7)$ & $7(50.0)$ & \\
\hline Both domains & $1(3.0)$ & $6(22.2)$ & & $4(8.7)$ & $3(21.4)$ & \\
\hline \multicolumn{7}{|l|}{ FBXW7status $^{d}$} \\
\hline Mutated & $6(20.0)$ & $7(29.2)$ & 0.434 & $11(27.5)$ & $2(14.3)$ & 0.320 \\
\hline WT & $24(80.0)$ & $17(70.8)$ & & $29(72.5)$ & $12(85.7)$ & \\
\hline Total & $33(100)$ & $27(100)$ & & $46(100)$ & $14(100)$ & \\
\hline
\end{tabular}

${ }^{a}$ insertions and/or deletions; WBC, white blood cells count; ${ }^{b}$ classification according to EGIL criteria, flow cytometry was not performed in 2 cases with mutations, both were classified as point/missense; ${ }^{c} \mathrm{CD} 10$ status was not available in 7 cases, being 5 with point mutations and 2 complex; and 6 classified as missense and 1 as nonsense; ${ }^{d}$ FBXW7 mutational status was not performed in 28 cases out of 138,22 of 28 were WT, 3 presented point mutations and 3 complex mutations, all 6 mutations were classified as missense

The results of the OS univariate analyses of T-ALL cases considering variables such as age, WBC at onset of disease, T-ALL cellular subtypes, CD10 expression, skin colour, response to induction therapy, mutational status of NOTCH1, FBXW7, NOTCH1-FBXW7 group, KRAS and PTEN, presence of SIL-TAL1 fusion, and ectopic expression of TLX3 are shown in Table 3, Figure 1 and Additional file 2: Figure S1.No statistically significant impact in OS was found for the following variables: age, WBC, T-ALL subtypes, CD10 expression, skin colour, mutational status of FBXW7, KRAS and PTEN, and ectopic expression of TLX3.

Test of equality of survival distribution for the differences of NOTCH1 status (mutated vs. wild-type) showed no statistical significant results $(53.3 \%$ vs. $48.7 \%$; $p=$ 0.479). On the other hand, carriers of NOTCH1 complex mutations (indels) exhibited a favourable OS when compared with carriers of point mutations $(66.7 \%$ vs. $42.4 \%$; $p=0.031 ;$ HR 2.33, CI95\% 1.05-5.18) (Figures 1B), NOTCH1 wild-type patients showed an intermediate OS rate $(48.7 \%)$. OS differences between complex and point NOTCH1 mutations were more marked in the patients treated with the BFM protocols backbone strategies (27.3\% vs. $62.5 \% ; p=0.041)$. For NOTCH1-FBXW7 combined mutations no impact on OS was observed (58.1\% vs. $47.9 \%)$, mutated in NOTCH1-FBXW7 vs. WT; $p=0.319$ showed in Figure 1C.

The presence of SIL-TAL1 fusion was predictive of a worse outcome (OS $37.8 \%$ vs. $56.5 \%$; $p=0.012$; HR 1.89, CI95\% 1.13-3.17) (Figure 1D). Multivariate Cox analysis showed that the type of NOTCH1 mutations (point vs. complex mutations) was not an independent prognostic factor $(p=0.769)$, compared with SIL-TAL1 fusion ( $p=0.04$; HR 2.87; 95\% CI 1.39-5.92) and induction response $(p=0.10$; HR 2.69; 95\% CI 1.27-5.71), even when adjusted by treatment protocol. 


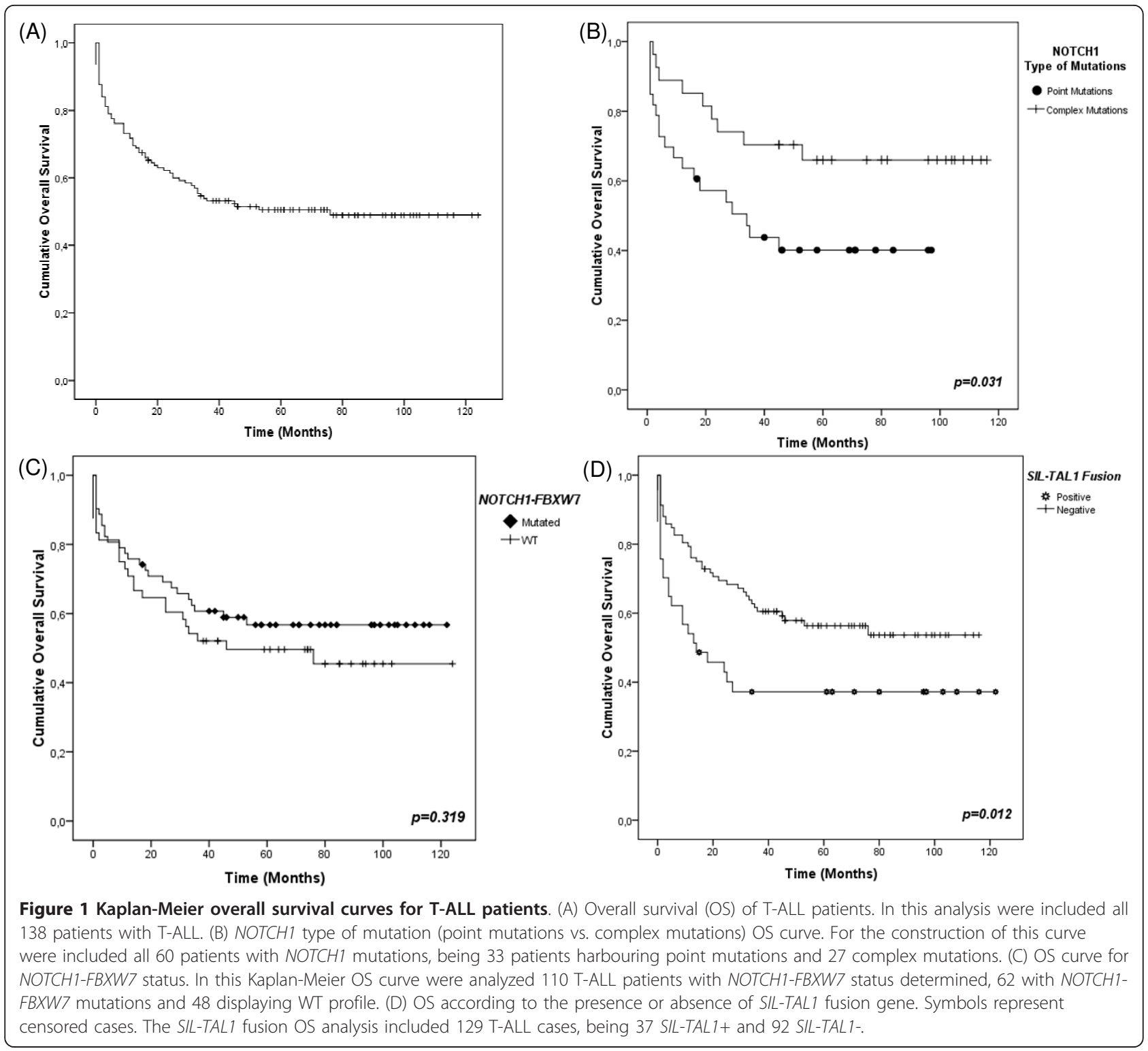

In 80 cases, the initial response to induction phase (day 30-33) was recorded, with $52.5 \%$ of cases exhibiting induction failure, independent on protocol allocation. Of the 38 cases that achieved clinical remission, 26 (72.5\%) maintained the initial response and $12(27.5 \%)$ relapsed during maintenance therapy and died. Of 42 cases with induction failure 11 (26.2\%) evolved to death and the remaining 31 were submitted to re-induction therapy (14 with secondary remission).

Induction therapy response was significantly associated with a longer overall survival, both in univariate and multivariate analyses $(p<0.0001$; HR 2.86 ; $95 \%$ CI 1.38 5.94) as shown in Table 3. However, no significant association was found between any of the molecular alterations and induction response (data not shown).
Ninety-five patients had sufficient clinical data for conducting EFS analysis (9 cases that evolved to death previously to treatment due to severity of disease were excluded of this analysis). All variables evaluated in the OS analysis were also included in EFS investigations. Results of EFS analysis are described in Table 3.

EFS exhibited no difference according to NOTCH1FBXW7 status (40.5\% Mutated, 48.5\% WT, $p=0.286$ ) (Figure 2A). For patients bearing complex NOTCH1 mutations the EFS was better than for those presenting point mutations $(30.4 \%, 40 \%$ respectively, $p=0.289$ ), albeit not statistically significant (Figure 2B). EFS was significantly worse in patients bearing SIL-TAL1 (18.2\% vs. $43.8 \% ; p=0.004$ ) (Figure $2 \mathrm{C}$ ), and also in those patients with no response to induction therapy $(32.4 \%$ 
Table 3 Univariate analysis of overall (OS) and event-free survival (EFS) in T-ALL patients

\begin{tabular}{|c|c|c|c|c|c|c|}
\hline Variables & $\operatorname{OS}^{a}(95 \% \mathrm{Cl})$ & $p^{\mathbf{b}}$ & HR $^{c}$ (Lower-Upper) & $\mathrm{EFS}^{\mathrm{a}}(95 \% \mathrm{Cl})$ & $p^{\mathrm{b}}$ & HR $^{c}$ (Lower-Upper) \\
\hline \multicolumn{7}{|l|}{ Age (Years) } \\
\hline$<10$ & $45.1(48.9-74.5)$ & 0.275 & $0.77(0.48-1.24)$ & $28.8(30.8-57.8)$ & 0.044 & $0.58(0.34-1.00)$ \\
\hline 10-18 & $56.7(60.7-88.0)$ & & & $51.2(51.9-85.8)$ & & \\
\hline \multicolumn{7}{|l|}{ WBC $\left(\times 10^{9} / L\right)$} \\
\hline$<50$ & $47.6(51.6-79.4)$ & 0.606 & $0.88(0.55-1.42)$ & $41.3(40.9-73.3)$ & 0.859 & $1.05(0.63-1.76)$ \\
\hline$\geq 50$ & $53.3(57.1-82.5)$ & & & $36.7(39.2-68.5)$ & & \\
\hline \multicolumn{7}{|l|}{ EGIL } \\
\hline $\mathrm{T}-\mathrm{I}$ & $33.3(28.8-77.2)$ & 0.411 & $0.93(0.74-1.18)$ & 31.3 (26.9-75.9) & 0.954 & $0.98(0.77-1.24)$ \\
\hline $\mathrm{T}-\|$ & $51.7(48.2-88.7)$ & & & 36.4 (31.5-75.5) & & \\
\hline$T-1 \| 1$ & $61.8(56.1-90.7)$ & & & 45.0 (32.9-78.6) & & \\
\hline T-IV & $51.0(44.0-70.9)$ & & & $36.7(30.2-60.9)$ & & \\
\hline \multicolumn{7}{|l|}{ CD10 } \\
\hline Positive & $52.8(50.6-86.9)$ & 0.696 & $1.12(0.63-1.97)$ & $48.1(42.6-83.6)$ & 0.097 & $1.67(0.89-3.08)$ \\
\hline Negative & $50.0(54.1-79.4)$ & & & $28.8(31.6-59.5)$ & & \\
\hline \multicolumn{7}{|l|}{ Skin colour } \\
\hline White & $55.0(54.5-81.5)$ & 0.451 & $1.20(0.74-1.96)$ & $33.3(32.1-61.5)$ & 0.331 & $0.78(0.46-1.30)$ \\
\hline Non-white & 47.4 (52.8-77.8) & & & $43.4(45.9-75.9)$ & & \\
\hline \multicolumn{7}{|l|}{ NOTCH1 } \\
\hline Mutated & $53.3(54.6-80.8)$ & 0.479 & $1.19(0.73-1.93)$ & $34.2(30.5-60.9)$ & 0.382 & $0.79(0.47-1.34)$ \\
\hline WT & $48.7(52.7-78.2)$ & & & $42.1(45.5-74.2)$ & & \\
\hline \multicolumn{7}{|c|}{ Type of mutation } \\
\hline Point & $42.4(32.4-61.9)$ & 0.031 & $0.43(0.19-0.95)$ & $30.4(19.1-51.4)$ & 0.289 & $0.65(0.28-1.47)$ \\
\hline Complex & $66.7(65.6-100.9)$ & & & $40.0(31.4-79.4)$ & & \\
\hline \multicolumn{7}{|l|}{ FBXW7 } \\
\hline Mutated & $61.9(54.8-102.3)$ & 0.512 & $1.28(0.60-2.73)$ & 58.3 (38.9-104.9) & 0.586 & $1.29(0.51-3.31)$ \\
\hline WT & $51.7(59.2-82.3)$ & & & $41.4(47.2-74.7)$ & & \\
\hline \multicolumn{7}{|l|}{ NOTCH1-FBXW7 } \\
\hline Mutated & $58.1(62.5-89.6)$ & 0.319 & $1.32(0.76-2.28)$ & $40.5(37.6-72.3)$ & 0.286 & $0.71(0.37-1.34)$ \\
\hline WT & 47.9 (50.7-82.3) & & & $48.5(51.1-87.7)$ & & \\
\hline \multicolumn{7}{|l|}{ SIL-TAL1 } \\
\hline Positive & $37.8(32.1-68.3)$ & 0.012 & $0.53(0.32-0.88)$ & $18.2(11.9-48.7)$ & 0.004 & $0.46(0.26-0.81)$ \\
\hline Negative & $56.5(60.9-81.9)$ & & & $43.8(46.4-71.1)$ & & \\
\hline \multicolumn{7}{|l|}{ HOX11L2 } \\
\hline Expressed & $40.0(13.4-44.6)$ & 0.469 & $0.74(0.32-1.71)$ & $50.0(14.3-50.2)$ & 0.806 & $1.14(0.41-3.16)$ \\
\hline Absent & $48.6(54.1-75.2)$ & & & $35.6(39.3-63.6)$ & & \\
\hline \multicolumn{7}{|l|}{ KRAS } \\
\hline Mutated & $60.0(32.7-78.1)$ & 0.585 & $1.32(0.48-3.67)$ & $57.1(21.4-79.7)$ & 0.584 & $1.38(0.43-4.46)$ \\
\hline WT & $49.5(55.7-77.9)$ & & & $36.9(41.5-66.9)$ & & \\
\hline \multicolumn{7}{|l|}{ PTEN } \\
\hline Mutated & $44.4(15.1-56.1)$ & 0.359 & $0.65(0.26-1.66)$ & $30.8(34.6-66.1)$ & 0.693 & $0.31(0.53-1.51)$ \\
\hline WT & $57.5(63.5-86.6)$ & & & $42.0(41.0-71.8)$ & & \\
\hline \multicolumn{7}{|c|}{ Induction Response ${ }^{d}$} \\
\hline Yes & $68.4(76.2-106.9)$ & $<0.001$ & $3.45(1.74-6.85)$ & $70.3(75.5-107.7)$ & $<0.001$ & $3.54(1.71-7.32)$ \\
\hline No & $33.3(25.9-55.9)$ & & & $32.4(23.4-48.6)$ & & \\
\hline
\end{tabular}

${ }^{\mathrm{a}}$ Survival in percent; ${ }^{\mathrm{b}}$ Mantel-Cox (log-rank) test; ${ }^{\mathrm{c}}$ last category used as reference; ${ }^{\mathrm{d}}$ evaluated in day $30-33$ of treatment; $H R$ hazard risk, $W T$ wild-type, $W B C$ white blood cells count

vs. 70.3\%, $p<0.0001$ ) (Figure 2D). Multivariate Cox analysis identified only SIL-TAL1 (HR 3.12; 95\% CI 1.48-6.57) and induction failure (HR 3.07; 95\% CI 1.44$6.55)$ as independent negative prognostic factors.

\section{Discussion}

In a previous analysis, we compared the presence of $\mathrm{CD}_{1 \mathrm{a}^{+\mathrm{ve}}}$ phenotype, SIL-TAL1 status, TLX3 abnormal expression and the clinical features on the impact in the 


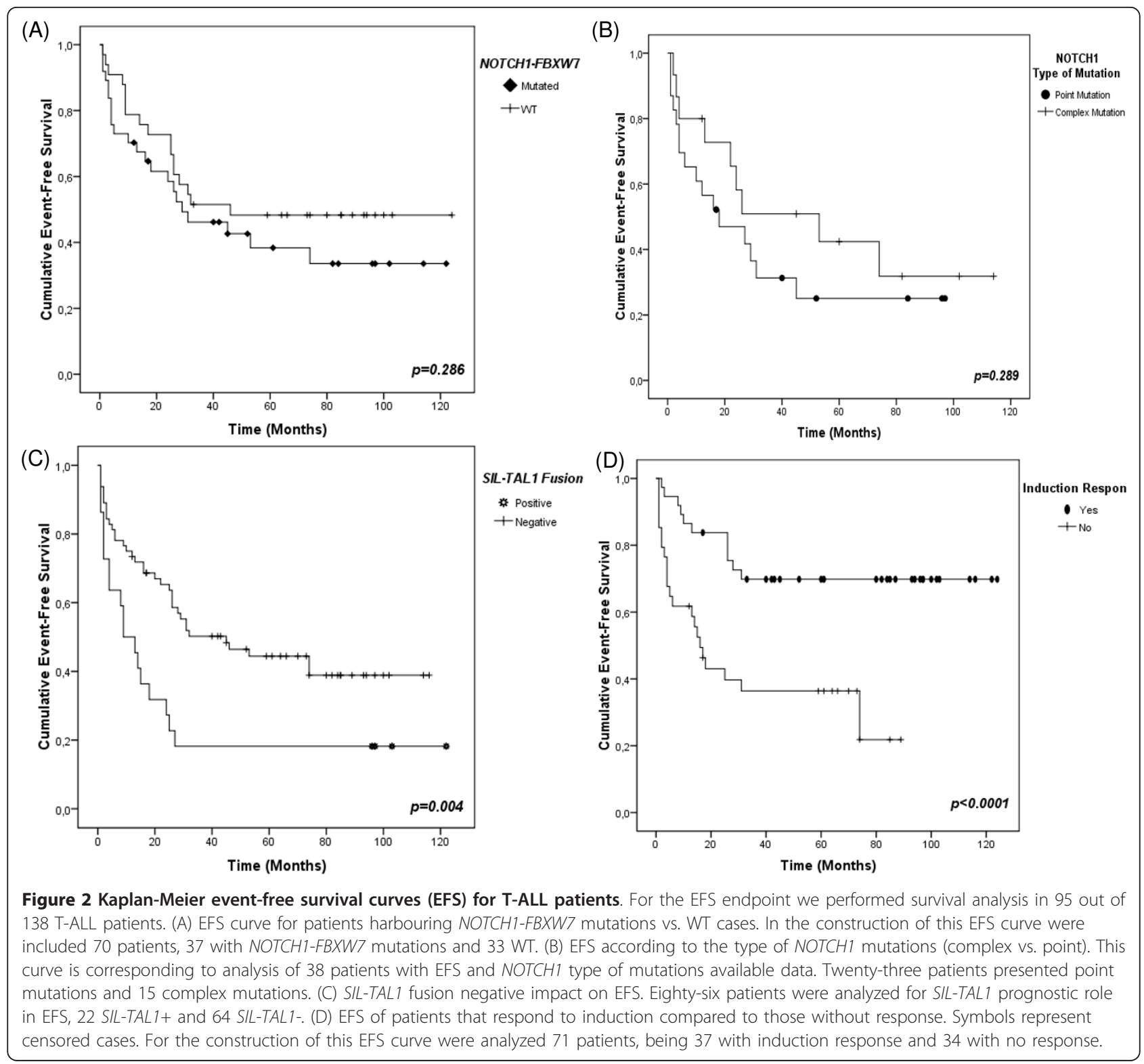

prognosis of T-ALL in series of Brazilian children and young adults [6]; whereas the presence of SIL-TAL1 had a poor outcome, particularly in younger children, either ectopic TLX3 or immunophenotyping were not predictive factors for outcome corroborating with data described by the Dutch Childhood Oncology Group [25]. Albeit, molecular alterations such as SIL-TAL1, $T L X 3$, and NOTCH1 mutations have been reported as T-ALL prognostic factors, due to scarce studies worldwide, most of them have inconsistent results $[3,10,25,26]$.

Investigation of NOTCH1 mutations and their relationships with $\mathrm{T}$-cell specific markers is still relevant because of its importance as a potential therapeutic target and prognostic marker [27]. In this context, the current study is one of the largest homogenous series of T-ALL childhood patients incorporating NOTCH1 mutation screening. Overall the NOTCH1 mutations were not correlated with variables considered as prognostic factors such as, age range, $\mathrm{T}$-cell subtypes or other molecular aberrations. However, new interesting information concerning the association between WBC count and type of NOTCH1 mutation was observed ( $p=$ 0.035). This finding cannot be compared with other data, since there is no similar analysis reported on the literature.

The frequency of NOTCH1 mutation in our series of patients was $43.5 \%$, which is consistent with the results of previously reported clinical series, that showed mutation frequencies between 31 and $62 \%$, depending on the 
series size and age groups $[3,9,25,28,29]$. The NOTCH1 mutation screening found 33 point-mutations and 27 complex-mutations. Fifty-three previously unreported unique NOTCH1 mutations were found in our series of Brazilian patients. In the NOTCH1 literature reports, the description of new mutations is a relatively frequent event [3,25,28-30]. To exclude false-positive results leading to the large number of new mutations, direct sequencing analyses were performed in different laboratory conditions and the same mutations were amplified with concordant results proving that contamination was unlikely. Moreover, NOTCH1 mutation profile concerning the domain affected (HD, PEST, or both) and type of mutations (point or complex) agreed with previously reported molecular analysis $[3,10]$.

Associations between NOTCH1 mutations and outcome have been inconsistent in different series of cases [7]. This discrepancy may be due to differences in the therapeutic protocols for each series. We have not found significance on prognosis for NOTCH1 status (mutated vs. WT) in our T-ALL cases, and this finding is in accordance to major T-ALL studies [9,25,28]. Additionally we found that complex mutations were associated with a longer survival time than point mutations ( $p=0.031)$, although the impact of mutation type did not stand as a prognostic factor for EFS. We believe that this lack of significance in the EFS analysis could be due to the small number of patients $(n=38)$ with EFS and NOTCH1 type of mutations available data. Then, we indicate the need to replicate this finding in an independent group of patients. Given the distinct functional effects of different mutations in NOTCH1 domains $[7,9]$, our results raises the issue of the potential differential role of $N O T C H 1$ mutation in the course of disease. Although no functional study has addressed this issue, we speculate that complex mutations would alter the Notch1 protein structure more drastically than point mutations, so blast cells would be more vulnerable to therapy responses. It is also possible that, as complex mutations were seen associated with high WBC, cases with complex mutations their presence lead to an initial more aggressive treatment, and hence to a sustained survival.

Key pathways related to Notch1 include the Ras/MAP Kinase and PI3-kinase/Akt. For the Ras/MAP Kinase pathway, since the majority of the studies evaluated the impact of KRAS mutations in mouse models with T-cell neoplasia [31,32], our KRAS data provides additional information in the evaluation of frequency and prognostic impact of these mutations in the largest described series of T-ALL patients. We found it in $9.5 \%$ of cases, without any impact on outcome. For the crosstalk between PI3-kinase/Akt and Notch1 pathways is described that Notch1 signalling via Hes 1 down- regulates PTEN, an important negative regulator of PI3kinase/Akt signalling [33]. Even with clear functional interaction between NOTCH1 and PTEN genes, no association for mutational status of these genes was found. Regarding the survival analysis, PTEN mutations displayed no statistically significance impact in OS or EFS (Table 3). Our results are in agreement with those of Gutierrez et al. [34], but are discordant with previous Brazilian results [24], which found a negative prognostic impact of PTEN mutations in T-ALLs. This variance may be due to the different classification of mutations (biallelic vs. monoallelic vs. wild-type) performed by Jotta et al. [24], indicating that the role of PTEN mutations as prognostic markers in T-ALL patients is not yet fully defined.

Mutations affecting $F B X W 7$ in leukemic cells mediate Notch1 pathway activation [35]. Since NOTCH1 and $F B X W 7$ mutations lead to the same effect, the hypothesis that T-ALL with FBXW7 mutations would be clinically similar to those with activating NOTCH1 mutations is reasonable [20]. Based on this idea, we performed a joint mutational analysis for these genes and our data of NOTCH1-FBXW7 mutation rate was $56.4 \%$ of T-ALLs, which is in line with previous reports on childhood T-ALL $[35,36]$. Concerning survival analysis, NOTCH1-FBXW7 mutational status showed no statistically significant impact on OS and EFS analysis. This absence of significance on OS and EFS analysis is concordant with other previously described results in representative series of paediatric T-ALL [19,20,37].

We have previously shown that $S I L-T A L 1$ was an agedependent prognostic factor, when rare cases of T-ALL in children with less than 1 year-old were included [6]. However, the present analysis excluding such young cases demonstrates that SIL-TAL1 is an independent adverse prognostic factor in older children as well.

\section{Conclusion}

In our series the status of NOTCH1 mutations alone or in combination with $F B X W 7$ did not impacted in $\mathrm{T}$ ALL prognosis. Nevertheless, NOTCH1 complex mutations were associated with a longer T-ALL overall survival, and SIL-TAL1 was validated as a marker of worse prognosis. These data should be tested in other series of cases to confirm the relevance of such analyses.

\section{Additional material}

\footnotetext{
Additional file 1: Table S1. Detailed descriptions of all NOTCH1 mutations sequences according to domain involved.

Additional file 2: Figure S1. Kaplan-Meier overall survival curves for TALL patients. (A) Overall survival (OS) according to the presence of KRAS mutations. One hundred and five cases were included in this analysis, 10 with KRAS mutated and $95 \mathrm{WT}$. (B) OS according to the presence of PTEN mutations. In the PTEN OS analysis were included 96 patients,
} 
being 9 with mutations and 87 WT. (C) OS according to the expression of the TLX3 gene. For the construction of this OS curve, 119T-ALL cases were analyzed, 10 TLX3+ and 109 TLX3-(D) OS of patients treated with GBTLI (Brazilian Group for Treatment of Childhood Leukaemia) therapeutic protocol compared with ALL-BFM (Berlin-Frankfurt-Munster) protocols backbone strategies. Symbols represent censored cases. All 138 cases were included for Treatment Protocol OS analysis, 101 treated according GBTLI and 37 according BFM.

\section{Acknowledgements}

We are grateful to paediatricians from different regions of Brazil who provided general and clinical data and to the children and parents who agreed to participate in this study. List of paediatricians from Brazilian Collaborative Study Group of Infant Acute Leukaemia that contributing as coauthors to the study: Jozina Maria Andrade Agareno $(n=10)$, Dora Márcia Santana Alencar $(n=10)$, Andrea Gadelha Nóbrega $(n=10)$, Tereza Cristina Cardoso Fonseca $(n=9)$, Anna Carolina Silva Dias $(n=8)$, Eloísa Cartaxo Eloy Fialho $(n=8)$, Alexandre Gustavo Apa $(n=7)$, Jane de Almeida Dobbin $(n=$ 7), Edinalva Pereira Leite $(n=7)$, Fernando Werneck $(n=7)$, Flávia Nogueira S. de Araújo $(n=6)$, Lilian Maria Burlacchini de Carvalho $(n=6)$, Maria Dolores Fonseca Dorea $(n=6)$, Silvia Rocha Caetano Agra $(n=5)$, Rosania Maria Basegio $(n=5)$, Gilson Guedes Filho $(n=5)$, Cynthia Curvello Neves ( $n$ =5), Mônica Lankszner $(n=5)$, Isis Maria Quezado Magalhães $(n=5)$, and Anelisa Schitini Costa $(n=5)$.

MSPO is supported in part by a grant-in-aid from CNPq (\#473124/2008-4; \#302423/2010-9) and INCT para Controle do Cancer, CNPq 573806/2008-0; FAPERJ E26/170.026/2008. MB Mansur is supported by the CAPES Foundation. JAY is supported by grant from Fundação de Amparo à Pesquisa do Estado de São Paulo (FAPESP, 2008/10034-1). RH is supported in part by the INCT para Controle do Cancer INCA program. JLW was supported by a Leukemia and Lymphoma Society Translational Research Grant 6026-10.

\section{Author details}

${ }^{1}$ Paediatric Haematology-Oncology Program, Research Centre, Instituto Nacional de Câncer, Rio de Janeiro, RJ, Brazil. 'Bone Marrow Transplantation Centre (CEMO), Instituto Nacional de Câncer, Rio de Janeiro, RJ, Brazil. ${ }^{3}$ Human Genome Studies Center, Instituto de Biociências, Universidade de São Paulo, São Paulo, SP, Brazil. ${ }^{4}$ Molecular Biology Laboratory, Centro Infantil Boldrini, Campinas, SP, Brazil. ${ }^{5}$ Laboratory for Molecular Epidemiology, Department of Epidemiology \& Biostatistics, University of California, San Francisco, CA, USA. ${ }^{6}$ Paediatric Haematology-Oncology Program, 6th floor, Research, Center, Instituto Nacional de Câncer-MS, Rua André Cavalcanti, 37, 20231-050 Rio de Janeiro, RJ, Brazil.

\section{Authors' contributions}

MBM designed, performed and wrote the paper. MBM, TCB and AS analysed the NOTCH1 and FBXW7 sequencing data. RH did all the statistical analyses. PYJ, JAY, RH and JLW helped with technical approaches, results discussions and data analyzes. MSPO designed and supervised the study and wrote the manuscript. All authors approved the manuscript descriptions.

\section{Competing interests}

The authors declare that they have no competing interests.

Received: 2 June 2011 Accepted: 6 January 2012

Published: 6 January 2012

\section{References}

1. Aifantis I, Raetz E, Buonamici S: Molecular pathogenesis of T-cell leukaemia and lymphoma. Nat Rev Immunol 2008, 8(5):380-390.

2. Pui CH, Robison LL, Look AT: Acute lymphoblastic leukaemia. Lancet 2008, 371(9617):1030-1043.

3. Breit S, Stanulla M, Flohr T, Schrappe M, Ludwig WD, Tolle G, Happich M, Muckenthaler MU, Kulozik AE: Activating NOTCH1 mutations predict favorable early treatment response and long-term outcome in childhood precursor T-cell lymphoblastic leukemia. Blood 2006, 108(4):1151-1157.
4. Ferrando AA, Neuberg DS, Dodge RK, Paietta E, Larson RA, Wiernik PH Rowe JM, Caligiuri MA, Bloomfield CD, Look AT: Prognostic importance of TLX1 (HOX11) oncogene expression in adults with T-cell acute lymphoblastic leukaemia. Lancet 2004, 363(9408):535-536.

5. Ferrando AA, Neuberg DS, Staunton J, Loh ML, Huard C, Raimondi SC, Behm FG, Pui CH, Downing JR, Gilliland DG, et al: Gene expression signatures define novel oncogenic pathways in T cell acute lymphoblastic leukemia. Cancer Cell 2002, 1(1):75-87.

6. Mansur MB, Emerenciano M, Brewer L, Sant'Ana M, Mendonca N, Thuler LC, Koifman S, Pombo-de-Oliveira MS: SIL-TAL1 fusion gene negative impact in T-cell acute lymphoblastic leukemia outcome. Leuk Lymphoma 2009, 50(8):1318-1325

7. Aster JC, Blacklow SC, Pear WS: Notch signalling in T-cell lymphoblastic leukaemia/lymphoma and other haematological malignancies. J Pathol 2011, 223(2):262-273.

8. Ellisen LW, Bird J, West DC, Soreng AL, Reynolds TC, Smith SD, Sklar J: TAN1 , the human homolog of the Drosophila notch gene, is broken by chromosomal translocations in T lymphoblastic neoplasms. Cell 1991, 66(4):649-661

9. Weng AP, Ferrando AA, Lee W, Morris JPt, Silverman LB, Sanchez-lrizarry $C$, Blacklow SC, Look AT, Aster JC: Activating mutations of NOTCH1 in human T cell acute lymphoblastic leukemia. Science 2004, 306(5694):269-271.

10. Grabher $\mathrm{C}$, von Boehmer H, Look AT: Notch 1 activation in the molecular pathogenesis of T-cell acute lymphoblastic leukaemia. Nat Rev Cancer 2006, 6(5):347-359.

11. Mullighan CG: Mutations of NOTCH1, FBXW7, and prognosis in T-lineage acute lymphoblastic leukemia. Haematologica 2009, 94(10):1338-1340.

12. Pombo de Oliveira MS, Koifman S, Vasconcelos GM, Emerenciano M, de Oliveira Novaes C: Development and perspective of current Brazilian studies on the epidemiology of childhood leukemia. Blood Cells Mol Dis 2009, 42(2):121-125.

13. World Health Organization Classification of Tumours: Pathology and Genetics of Tumours of Haematopoietic and Lymphoid Tissues. In International Agency for Research on Cancer -IARC, 2001. Edited by: Jaffe ES, Harris NL, Stein H, Vardiman JW. Lyon: IARC Press; 2001:

14. World Health Organization Classification of Tumours of Haematopoietic and Lymphoid Tissues. Edited by: Swerdlow SH, Campo E, Harris NL, Jaffe ES, Pileri SA, Stein H, Thiele J, Vardiman JW. Lyon: IARC; 2008:

15. Parra FC, Amado RC, Lambertucci JR, Rocha J, Antunes CM, Pena SD: Color and genomic ancestry in Brazilians. Proc Natl Acad Sci USA 2003, 100(1):177-182.

16. Brandalise SR, Pinheiro VR, Aguiar SS, Matsuda El, Otubo R, Yunes JA, Pereira WV, Carvalho EG, Cristofani LM, Souza MS, et al: Benefits of the intermittent use of 6-mercaptopurine and methotrexate in maintenance treatment for low-risk acute lymphoblastic leukemia in children: randomized trial from the Brazilian childhood cooperative groupprotocol ALL-99. J Clin Oncol 2010, 28(11):1911-1918.

17. Moricke A, Zimmermann M, Reiter A, Henze G, Schrauder A, Gadner H, Ludwig WD, Ritter J, Harbott J, Mann G, et al: Long-term results of five consecutive trials in childhood acute lymphoblastic leukemia performed by the ALL-BFM study group from 1981 to 2000. Leukemia 2010, 24(2):265-284.

18. Bene MC, Castoldi G, Knapp W, Ludwig WD, Matutes E, Orfao A, van't Veer MB: Proposals for the immunological classification of acute leukemias. European Group for the Immunological Characterization of Leukemias (EGIL). Leukemia 1995, 9(10):1783-1786.

19. Clappier E, Collette S, Grardel N, Girard S, Suarez L, Brunie G, Kaltenbach S, Yakouben K, Mazingue F, Robert A, et al: NOTCH1 and FBXW7 mutations have a favorable impact on early response to treatment, but not on outcome, in children with T-cell acute lymphoblastic leukemia (T-ALL) treated on EORTC trials 58881 and 58951. Leukemia 2010, 24(12):2023-2031.

20. Kox C, Zimmermann M, Stanulla M, Leible S, Schrappe M, Ludwig WD, Koehler R, Tolle G, Bandapalli OR, Breit $\mathrm{S}$, et al: The favorable effect of activating NOTCH1 receptor mutations on long-term outcome in T-ALL patients treated on the ALL-BFM 2000 protocol can be separated from FBXW7 loss of function. Leukemia 2010, 24(12):2005-2013.

21. Bernard OA, Busson-LeConiat M, Ballerini P, Mauchauffe M, Della Valle $V$, Monni R, Nguyen Khac F, Mercher T, Penard-Lacronique V, Pasturaud P, et al: A new recurrent and specific cryptic translocation, $\mathrm{t}(5 ; 14)(\mathrm{q} 35 ; \mathrm{q} 32)$, 
is associated with expression of the Hox11L2 gene in T acute lymphoblastic leukemia. Leukemia 2001, 15(10):1495-1504.

22. van Dongen JJ, Macintyre EA, Gabert JA, Delabesse E, Rossi V, Saglio G, Gottardi E, Rambaldi A, Dotti G, Griesinger F, et al: Standardized RT-PCR analysis of fusion gene transcripts from chromosome aberrations in acute leukemia for detection of minimal residual disease. Report of the BIOMED-1 concerted action: investigation of minimal residual disease in acute leukemia. Leukemia 1999, 13(12):1901-1928.

23. Bornholdt J, Hansen J, Steiniche T, Dictor M, Antonsen A, Wolff $H$, Schlunssen V, Holmila R, Luce D, Vogel U, et al: K-ras mutations in sinonasal cancers in relation to wood dust exposure. BMC Cancer 2008, 8:53.

24. Jotta PY, Ganazza MA, Silva A, Viana MB, da Silva MJ, Zambaldi LJ, Barata JT, Brandalise SR, Yunes JA: Negative prognostic impact of PTEN mutation in pediatric T-cell acute lymphoblastic leukemia. Leukemia 2010, 24(1):239-242.

25. van Grotel M, Meijerink JP, van Wering ER, Langerak AW, Beverloo HB, BuijsGladdines JG, Burger NB, Passier M, van Lieshout EM, Kamps WA, et al: Prognostic significance of molecular-cytogenetic abnormalities in pediatric T-ALL is not explained by immunophenotypic differences. Leukemia 2008, 22(1):124-131.

26. Meijerink JP: Genetic rearrangements in relation to immunophenotype and outcome in T-cell acute lymphoblastic leukaemia. Best Pract Res Clin Haematol 2010, 23(3):307-318.

27. Paganin $\mathrm{M}$, Ferrando A: Molecular pathogenesis and targeted therapies for NOTCH1-induced T-cell acute lymphoblastic leukemia. Blood Rev 2011, 25(2):83-90.

28. Larson Gedman A, Chen Q, Kugel Desmoulin S, Ge Y, LaFiura K, Haska CL, Cherian C, Devidas M, Linda SB, Taub JW, et al: The impact of NOTCH1, FBW7 and PTEN mutations on prognosis and downstream signaling in pediatric T-cell acute lymphoblastic leukemia: a report from the Children's Oncology Group. Leukemia 2009, 23(8):1417-1425.

29. Park MJ, Taki T, Oda M, Watanabe T, Yumura-Yagi K, Kobayashi R, Suzuki N, Hara J, Horibe K, Hayashi Y: FBXW7 and NOTCH1 mutations in childhood $T$ cell acute lymphoblastic leukaemia and T cell non-Hodgkin lymphoma. Br J Haematol 2009, 145(2):198-206.

30. Zhu YM, Zhao WL, Fu JF, Shi JY, Pan Q, Hu J, Gao XD, Chen B, Li JM, Xiong SM, et al: NOTCH1 mutations in T-cell acute lymphoblastic leukemia: prognostic significance and implication in multifactorial leukemogenesis. Clin Cancer Res 2006, 12(10):3043-3049.

31. Dail M, Li Q, McDaniel A, Wong J, Akagi K, Huang B, Kang HC, Kogan SC, Shokat K, Wolff L, et al: Mutant Ikzf1, KrasG12D, and Notch1 cooperate in $\mathrm{T}$ lineage leukemogenesis and modulate responses to targeted agents. Proc Natl Acad Sci USA 2010, 107(11):5106-5111.

32. Kindler T, Cornejo MG, Scholl C, Liu J, Leeman DS, Haydu JE, Frohling S, Lee BH, Gilliland DG: K-RasG12D-induced T-cell lymphoblastic lymphoma/ leukemias harbor Notch1 mutations and are sensitive to gammasecretase inhibitors. Blood 2008, 112(8):3373-3382.

33. Palomero T, Sulis ML, Cortina M, Real PJ, Barnes K, Ciofani M, Caparros E, Buteau J, Brown K, Perkins SL, et al: Mutational loss of PTEN induces resistance to NOTCH1 inhibition in T-cell leukemia. Nat Med 2007, 13(10):1203-1210.

34. Gutierrez A, Sanda T, Grebliunaite R, Carracedo A, Salmena L, Ahn Y, Dahlberg S, Neuberg D, Moreau LA, Winter SS, et al: High frequency of PTEN, PI3K, and AKT abnormalities in T-cell acute lymphoblastic leukemia. Blood 2009, 114(3):647-650

35. O'Neil J, Grim J, Strack P, Rao S, Tibbitts D, Winter C, Hardwick J, Welcker M, Meijerink JP, Pieters $R$, et al: FBW7 mutations in leukemic cells mediate NOTCH pathway activation and resistance to gamma-secretase inhibitors. J Exp Med 2007, 204(8):1813-1824.

36. Thompson BJ, Buonamici S, Sulis ML, Palomero T, Vilimas T, Basso G, Ferrando A, Aifantis I: The SCFFBW7 ubiquitin ligase complex as a tumor suppressor in T cell leukemia. J Exp Med 2007, 204(8):1825-1835.

37. Zuurbier L, Homminga I, Calvert V, te Winkel ML, Buijs-Gladdines JG, Kooi C, Smits WK, Sonneveld E, Veerman AJ, Kamps WA, et al: NOTCH1 and/or FBXW7 mutations predict for initial good prednisone response but not for improved outcome in pediatric T-cell acute lymphoblastic leukemia patients treated on DCOG or COALL protocols. Leukemia 2010, 24(12):2014-2022.

\section{Pre-publication history}

The pre-publication history for this paper can be accessed here: http://www.biomedcentral.com/1471-2407/12/9/prepub

doi:10.1186/1471-2407-12-9

Cite this article as: Mansur et al:: Impact of complex NOTCH1 mutations on survival in paediatric T-cell leukaemia. BMC Cancer 2012 12:9.

\section{Submit your next manuscript to BioMed Central and take full advantage of:}

- Convenient online submission

- Thorough peer review

- No space constraints or color figure charges

- Immediate publication on acceptance

- Inclusion in PubMed, CAS, Scopus and Google Scholar

- Research which is freely available for redistribution

Submit your manuscript at www.biomedcentral.com/submit
C Biomed Central 